\title{
Perfil dos pacientes de hanseníase: uma revisão de literatura
}

\author{
Profille of leprosy patients: a literature review \\ Perfil de los pacientes con lepra: una revisión de la literatura
}

Recebido: 22/11/2021 | Revisado: 01/12/2021 | Aceito: 02/12/2021 | Publicado: 12/12/2021

Lys Vitória Ribeiro de Almeida

ORCID: https://orcid.org/0000-0003-3187-9372

Fundação Universidade Federal de Rondônia, Brasil

E-mail: lys.vitoria.trabalho@gmail.com

Renato Jesus de Oliveira

ORCID: https://orcid.org/0000-0002-4051-1919

Fundação Universidade Federal de Rondônia, Brasil

E-mail: renatoolivheira@gmail.com

Vitoria Morais de Oliveira

ORCID: https://orcid.org/0000-0003-1402-8330

Fundação Universidade Federal de Rondônia, Brasil

E-mail: vitoriavmo@hotmail.com

Janne Cavalcante Monteiro

ORCID: https://orcid.org/0000-0002-3677-4791

Fundação Universidade Federal de Rondônia, Brasil

E-mail: jannemonteiro@unir.br

Nathalia Halax Orfão

ORCID: https://orcid.org/0000-0002-8734-3393

Fundação Universidade Federal de Rondônia, Brasil

E-mail: nathaliahalax@unir.br

\begin{abstract}
Resumo
Objetivo: Analisar o perfil dos pacientes com hanseníase que são atendidos pelo Sistema Único de Saúde brasileiro. Metodologia: Trata-se de uma revisão integrativa de literatura, realizada nas bases de dados Web of Science, U. S. National Library of Medicine (PubMed) e Literatura Latino-Americana e do Caribe em Ciências da Saúde (LILACS), a partir da seleção de descritores indexados e seus respectivos sinônimos, os quais combinados por meio dos operadores booleanos AND e OR constituíram a expressão de busca, representando a pergunta norteadora. Foram incluídos artigos completos, publicados entre 2010 e 2021 em inglês, português ou espanhol. Resultados: Foram encontradas 73 publicações científicas, das quais após aplicados os critérios, foram selecionadas 13 para leitura na íntegra. Destes, nove artigos foram considerados para este estudo, cujo perfil dos pacientes com hanseníase se caracterizava com idade entre 37 e 49,28 anos, sexo masculino, residentes na área urbana, ensino primário incompleto, aposentados e donas de casa que viviam com valor entre um e três salários mínimos, casos novos que em sua maioria foram encaminhados de outros serviços, formas clínicas limítrofe, dimórfica, não classificado e indeterminada, paucibacilar e grau de incapacidade física zero. Conclusão: Diante destes achados, este estudo permitiu identificar o perfil epidemiológico majoritário dos pacientes acometidos pela hanseníase no Brasil, reforçando sobre a importância da articulação dos serviços, manejo adequado dos casos e efetividade das ações de vigilância em saúde, cujo protagonismo deve ser realizado pela Atenção Primária à Saúde.
\end{abstract}

Palavras-chave: Hanseníase; Perfil de saúde; Sistema Único de Saúde; Brasil.

\begin{abstract}
Objective: To analyze the profile of patients with leprosy who are treated by the Brazilian Unified Health System. Methodology: This is an integrative literature review, carried out in the Web of Science, US National Library of Medicine (PubMed) and Latin American and Caribbean Literature in Health Sciences (LILACS) databases, from the selection of indexed descriptors and their synonyms, which combined through the Boolean operators AND and OR constituted the search expression, representing the guiding question. Full articles published between 2010 and 2021 in English, Portuguese or Spanish were included. Results: 73 scientific publications were found, from the information on the criteria, 13 were selected for full reading. Nine of these articles were considered for this study which profile of leprosy patients is aged between 37 and 49.28 years, male, urban residents, incomplete primary education, retirees and housewives who lived with a value between one and three algorithms, new cases that were mostly referred from other services, borderline clinical forms, dimorphic, unclassified and indeterminate, paucibacillary and zero degree of physical disability. Conclusion: In view of these findings, this study identified the epidemiological profile of the majority of patients affected by leprosy in Brazil, stressing the importance of articulation of services, adequate case management and effectiveness of health surveillance actions, whose role should be played by the Primary Health Care. Keywords: Leprosy; Health profile; Unified Health System; Brazil.
\end{abstract}




\begin{abstract}
Resumen
Objetivo: Analizar el perfil de los pacientes leprosos atendidos por el Sistema Único de Salud en Brasil. Metodología: Se trata de una revisión integradora de la literatura, realizada en las bases de datos Web of Science, National Library of EE Medicine. UU. (PubMed) y Literatura Latinoamericana y Caribeña en Ciencias de la Salud (LILACS), a partir de la selección de descriptores indexados y sus sinónimos, que se combinaron a través de los operadores booleanos Y y La constitución de la expresión de búsqueda, guía expresión la pregunta. Los artículos completos publicados entre 2010 y 2021 se incluyen en inglés, portugués o español. Resultados: Se encontraron 73 publicaciones científicas, con base en la información de los criterios, 13 fueron seleccionadas para lectura completa. De estos, se considerarán nuevos artículos para este estudio, cuyo perfil de pacientes leprosos se encuentra entre 37 y 49,28 años, varones, residentes urbanos, educación primaria incompleta, jubilados y amas de casa que vivido con un valor entre uno y tres algoritmos, nuevo. casos que se derivaron principalmente de otros servicios, formas clínicas limítrofes, dimórficas, no clasificadas e indeterminadas, paucibacilares y cierto grado de discapacidad física. Conclusión: A la luz de estos hechos, este estudio debe identificar el mayor perfil epidemiológico de los pacientes afectados por lepra en Brasil, destacando la importancia de articular los servicios, el adecuado manejo de los casos y la efectividad de las acciones de vigilancia en salud, cuyo protagonismo debe ser realizado por la Primaria Cuidado de la salud.
\end{abstract}

Palabras clave: Lepra; Perfil de salud; Sistema único de Salud; Brasil.

\title{
1. Introdução
}

A hanseníase é uma doença infectocontagiosa, de caráter crônico, causada pelo Mycobacterium leprae, a qual afeta a pele e os nervos periféricos causando lesões cutâneas e o comprometimento dos nervos (Brasil, 2008) de forma lenta e progressiva, podendo levar à incapacidade física. Seus sintomas incluem lesões, dormência e alterações na sensibilidade da pele (Brasil, 2017). Mundialmente, em 2020, foram contabilizados 127.396 novos casos e 129.192 registrados, o que resultou em uma taxa de detecção de 16,4 casos/por milhão de hab. e um coeficiente de prevalência de 16,6 casos/ por milhão de habitante, resultando em uma tendência crescente nos países em desenvolvimento e a sua inserção entre os Objetivos do Desenvolvimento do Milênio (WHO, 2021).

No Brasil, é uma doença de notificação compulsória no Sistema de Informação de Agravos de Notificação (SINAN) em todo território nacional, visando o monitoramento e a efetivação de medidas de prevenção e controle de vigilância em saúde, considerando a influência genética e imunidade individual contra o bacilo (Brasil, 2019; 2021a). O país ocupa o segundo dentre os 23 na tendência de detecção de casos novos de hanseníase, entre 2011 a 2020, o que enfatiza o grau de relevância mesmo diante dos avanços nas últimas décadas (WHO, 2021), incluindo a Estratégia Nacional para o Enfrentamento da Hanseníase (2019-2022) (Brasil, 2021b).

Esse cenário é agravado pelos diversos desafios e obstáculos que o sistema de saúde brasileiro ainda enfrenta na detecção precoce e início imediato do tratamento. Esses devem ser enfrentados de maneira que permitam a eliminação das barreiras que impedem o acesso da população aos serviços de saúde, interrupção da cadeia de transmissão, adesão à terapia e redução do estigma da doença, conforme o proposto pela Estratégia Global pela Hanseníase (WHO, 2016). Sendo assim, todos os esforços como compromisso político, ações estratégicas e o estabelecimento de parcerias eficazes e sólidas, visando a redução da carga da doença e das incapacidades físicas, são necessários para o enfrentamento dessa doença (Brasil, 2019).

Ao mesmo tempo é importante a integração e articulação, inclusive com comunicação, das ações de prevenção e controle da doença entre os diferentes serviços que constituem a Rede de Atenção à Saúde (RAS), haja vista que o modelo para controle de endemias no Brasil baseia-se no precoce diagnóstico, prevenção e tratamento dos casos (Santos et al., 2018), tornando-se essencial o fortalecimento e amplificação da busca ativa de casos (Brasil, 2021a).

Assim, este estudo teve como objetivo analisar o perfil dos pacientes com hanseníase que são atendidos pelo Sistema Único de Saúde brasileiro, de acordo com a literatura. 


\section{Metodologia}

Trata-se de uma revisão integrativa da literatura, na qual foi utilizada a estratégia PICo (The Joanna Briggs Institute, 2014) como método de construção da pergunta de pesquisa - "Qual o perfil de pacientes com hanseníase atendidos pelo sistema de saúde brasileiro?" -, onde o "P" se refere aos pacientes com hanseníase, o "I" ao perfil de saúde e o "Co" ao sistema de saúde brasileiro.

O levantamento e a seleção dos descritores indexados e seus respectivos sinônimos ocorreu nos Descritores em Ciências da Saúde (DeCS) e no Medical Subject Headings (MeSH) e quando combinados pelos operadores booleanos AND e OR constituíram a expressão de busca (Quadro 1).

Quadro 1 - Expressão de busca utilizado nesta revisão de literatura, 2021.

\begin{tabular}{|c|c|c|}
\hline Acrônimo & Conteúdo & Expressão de busca \\
\hline $\mathrm{P}$ & $\begin{array}{l}\text { Pacientes com } \\
\text { hanseníase }\end{array}$ & $\begin{array}{l}\text { Pacientes OR Patients OR Pacientes OR Clientes OR Doente OR Doentes OR Enferma OR Enfermo OR Paciente } \\
\text { OR "Pessoa Doente" OR "Pessoa Enferma" OR "Pessoa com Doença" OR "Pessoa com Enfermidade" OR } \\
\text { "Pessoas Doentes" OR "Pessoas Enfermas" OR "Pessoas com Doenças" OR "Pessoas com Enfermidades" OR } \\
\text { Client OR Clients OR Patient OR Enfermos OR "Persona Enferma" OR "Persona con Enfermedad" OR } \\
\text { "Personas Enfermas" OR "Personas con Enfermedades") AND (Hanseníase OR Leprosy OR Lepra OR "Doença } \\
\text { de Hansen" OR Lepra OR "Disease, Hansen" OR "Disease, Hansen's" OR "Hansen Disease" OR "Hansen's } \\
\text { Disease" OR "Hansens Disease" OR Leprosies OR "Enfermedad de Hansen" OR "Mal de Hansen" }\end{array}$ \\
\hline I & Perfil de saúde & $\begin{array}{l}\text { "Perfil de Saúde" OR "Health Profile" OR "Perfil de Salud" OR "Perfil Epidemiológico" OR "Perfis } \\
\text { Epidemiológicos" OR "Epidemiological Profile" OR "Epidemiological Profiles" OR "Perfil Epidemiológico" } \\
\text { OR "Perfil del Área" OR "Perfiles Epidemiológicos" OR "Monitoramento Epidemiológico" OR } \\
\text { "Epidemiological Monitoring" OR "Monitoreo Epidemiológico" OR "Monitoração Epidemiológica" OR } \\
\text { "Monitorização Epidemiológica" OR "Vigilância Epidemiológica" OR "Epidemiologic Monitoring" OR } \\
\text { "Epidemiologic Surveillance" OR "Epidemiologic Surveillances" OR "Monitoring, Epidemiologic" OR } \\
\text { "Monitoring, Epidemiological" OR "Surveillance, Epidemiologic" OR "Surveillances, Epidemiologic" OR } \\
\text { "Monitorización Epidemiológica" OR "Vigilancia Epidemiológica" }\end{array}$ \\
\hline Co & $\begin{array}{l}\text { Sistema de } \\
\text { saúde } \\
\text { brasileiro }\end{array}$ & $\begin{array}{l}\text { "Sistemas de Saúde" OR "Health Systems" OR "Sistemas de Salud" OR "Sistema de Saúde" OR "Health } \\
\text { System" OR "Sistema de Salud" OR "Health Services" OR "Health Service" OR "Services, Health" OR } \\
\text { "Serviços de Saúde" OR "Servicios de Salud" OR "Consumo de Serviços de Saúde" OR "Rede Prestadora de } \\
\text { Serviços de Saúde" OR "Serviço de Saúde" OR "Serviços de Atenção ao Paciente" OR "Uso de Serviços de } \\
\text { Saúde" OR "Consumo de Servicios de Salud" OR "Servicio de Salud" OR "Servicios de Atención al Paciente" } \\
\text { OR "Uso de Servicios de Salud" OR "Utilización de Servicios de Salud" OR "Public Health" OR "Health, } \\
\text { Public" OR "Community Health" OR "Health, Community" OR "Environment, Preventive Medicine and Public } \\
\text { Health" OR "Environment, Preventive Medicine and Public Health" OR "Environment, Preventive Medicine \& } \\
\text { Public Health" OR "Health, Community" OR "Saúde Pública" OR "Meio Ambiente, Medicina Preventiva e } \\
\text { Saúde Pública" OR "Saúde Coletiva" OR "Saúde Comunitária" OR "Saúde da Comunidade" OR "Saúde das } \\
\text { Comunidades" OR "Salud Pública" OR "Ambiente, Medicina Preventiva y Salud Pública" OR "Medio } \\
\text { Ambiente, Medicina Preventiva y Salud Pública" OR "Salud Colectiva" OR "Salud Comunitaria" OR "Salud de } \\
\text { la Comunidad" OR "Salud de las Comunidades" OR "Sistema Unico de Saúde" OR SUS OR "Unified Health } \\
\text { System" OR "Brazilian Unified Health System" OR "Brazilian Unified National Health System" OR "Single } \\
\text { Health Care System" OR "Single Health System" OR "Unified Health Care System" OR "Sistema Unico de } \\
\text { Salud" }\end{array}$ \\
\hline
\end{tabular}

Fonte: Elaborado pelos autores (2021).

A busca foi realizada, em abril de 2021, nas bases de dados do Web of Science, U. S. National Library of Medicine (PubMed) e Literatura Latino-Americana e do Caribe em Ciências da Saúde (LILACS) por meio do acesso à Comunidade Acadêmica Federada (CAFe), no portal de periódicos da Coordenação de Aperfeiçoamento de Pessoal de Nível Superior (Capes). Como critérios de inclusão foram considerados os artigos completos publicados em português, espanhol ou inglês entre 2010 e 2021. Como exclusão, os materiais duplicados, monografias e resumos. O material selecionado foi exportado para o Rayyan (Ouzzani et al., 2016), onde foram excluídas as duplicidades e analisados o título e o resumo por três pesquisadores independentes, considerando o critério de elegibilidade, ou seja, estudos que abordavam sobre o perfil dos pacientes de hanseníase no sistema de saúde brasileiro. 
Posteriormente, os resultados foram apresentados em um quadro para melhor visualização dos achados encontrados, considerando o título, autor, ano de publicação, delineamento do estudo, objetivo, local de realização e os principais achados.

\section{Resultados}

Das 73 publicações encontradas nas bases de dados, 40 foram excluídas ainda na base de busca, considerando que não eram artigos completos (13), idioma (1) e ano de publicação anterior a 2010 (14); e no Rayyan os duplicados (12). Na segunda etapa da seleção foram excluídos 20 artigos por não atenderem ao critério de elegibilidade, uma vez que abordavam o perfil clínico de consultas dermatológicas relacionadas a acne, fotoenvelhecimento, câncer de pele não melanoma e ceratose actínica, tuberculose, câncer, filariose linfática, quimioprofilaxia e os que eram revisões bibliográficas ou analisavam dados de outro país (Índia e Colômbia). Totalizando 13 artigos para leitura na íntegra, onde quatro ainda foram excluídos, pois se caracterizavam como resumos simples (1) ou expandidos (3), sendo selecionado para esta revisão nove artigos (Figura 1).

Figura 1 - Fluxograma do processo de seleção dos artigos para esta revisão, 2021.

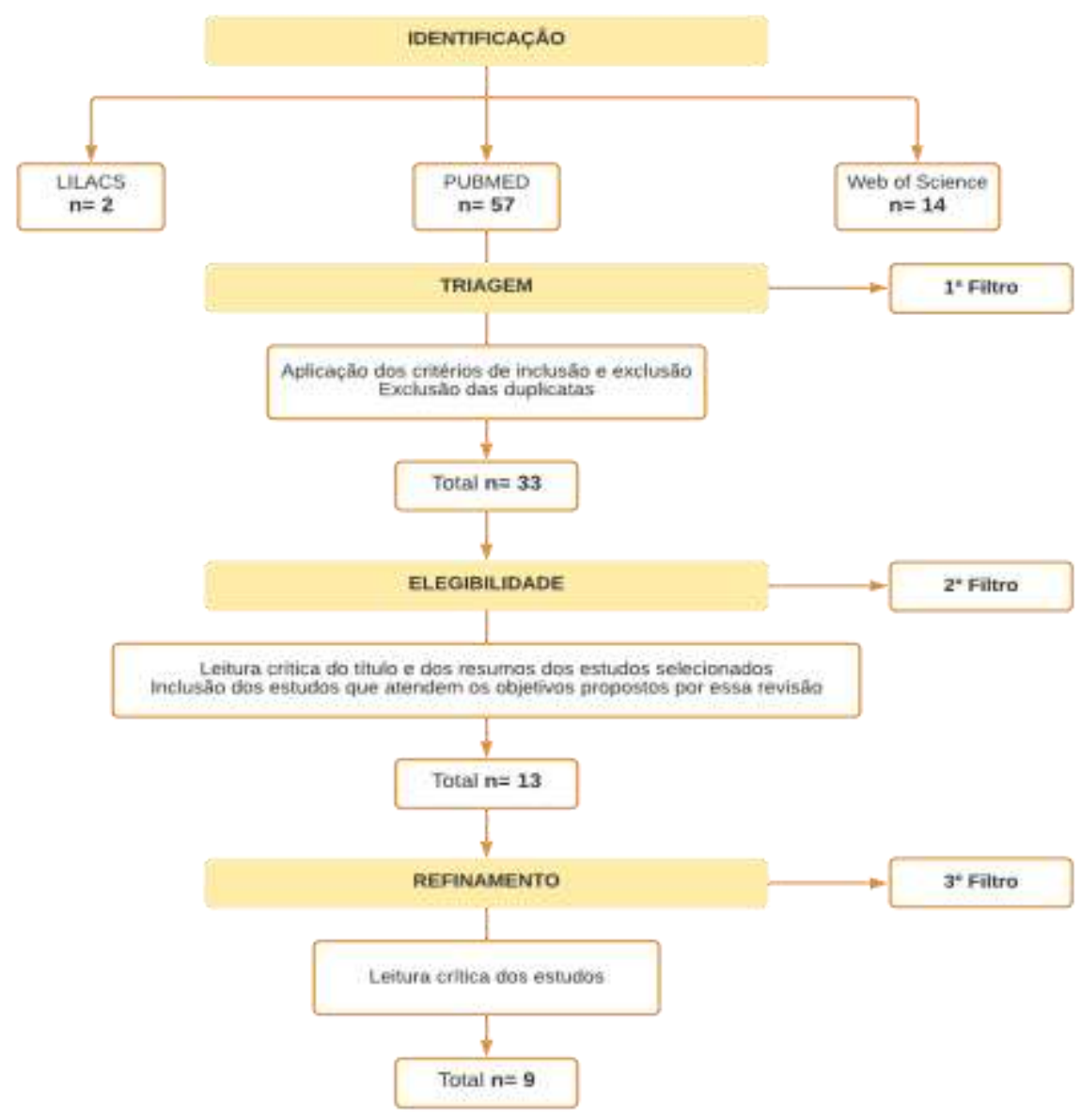

Fonte: Elaborado pelos autores (2021).

A maioria dos artigos foram publicados em 2020 (Melo et al., 2020; Santos et al., 2020), com delineamento transversal (Alves et al., 2017; Gaudenci et al., 2018; Melão et al., 2011; Melo et al., 2020; Queiroz et al., 2015; Santos et al., 2020) e retrospectivo (Araújo et al., 2021; Melão et al., 2011; Miranzi et al., 2010). Outrossim, as pesquisas tinham como objetivo identificar o perfil dos pacientes de hanseníase nos seus respectivos locais de estudo, principalmente no estado do Piauí (Alves et al., 2017; Melo et al., 2020) (Quadro 2). 
Quadro 2 - Caracterização dos artigos selecionados para esta revisão de literatura, de acordo com o título, autor, ano de publicação, objetivo, delineamento do estudo, local de realização e principais achados, 2021.

\begin{tabular}{|c|c|c|c|c|}
\hline AUTOR/ANO & OBJETIVO & $\begin{array}{c}\text { DELINEAMENTO } \\
\text { DO ESTUDO }\end{array}$ & LOCAL & PRINCIPAIS ACHADOS \\
\hline $\begin{array}{l}\text { Miranzi; Pereira; } \\
\text { Nunes, } 2010\end{array}$ & $\begin{array}{l}\text { Descrever o perfil epidemiológico da } \\
\text { população com diagnóstico de hanseníase, } \\
\text { no município de Uberaba, Estado de Minas } \\
\text { Gerais, Brasil, no período de } 2000 \text { a } 2006\end{array}$ & Estudo retrospectivo & $\begin{array}{l}\text { Uberaba, Estado } \\
\text { de Minas Gerais }\end{array}$ & $\begin{array}{l}\text { A detecção dos } 455 \text { casos de hanseníase registrados no período foi feita majoritariamente por } \\
\text { encaminhamento }(55,6 \%) \text {. Os pacientes eram, em sua maioria, do sexo masculino }(55,4 \%) \text {, pardos } \\
(33,4 \%) \text {, residiam na zona urbana }(82,8 \%) \text {, tinham média de quatro a sete anos de estudo }(34,9 \%) \text {, faixa } \\
\text { etária entre } 35 \text { a } 49 \text { anos }(31,4 \%) \text {. As variáveis clínicas mostram que } 31,4 \% \text { apresentavam incapacidade } \\
\text { grau zero e } 29,9 \% \text { tinham mais do que cinco lesões e } 29,7 \% \text { uma única lesão. Além disso, a maior parte } \\
\text { dos pacientes foram curados }(83,9 \%) \text {, apresentavam a forma clínica dimorfa }(69,1 \%) \text { e multibacilar } \\
(87,1 \%) \text {, com incapacidade ignorada ou em branco, } 33,8 \% \text { delas tiveram mais de } 5 \text { lesões e } 41,2 \% \text { dos } \\
\text { acometidos por incapacidade de grau II não possuíam nenhuma lesão. }\end{array}$ \\
\hline Melão et al., 2011 & $\begin{array}{l}\text { Fazer uma análise epidemiológica dos } \\
\text { portadores de hanseníase atendidos no } \\
\text { programa de hanseníase das onze cidades } \\
\text { integrantes da Associaçãa dos Municípios } \\
\text { da Região Carbonífera (AMREC), durante } \\
\text { o período de } 1^{\circ} \text { de janeiro de } 2001 \text { a } 31 \text { de } \\
\text { dezembro de } 2007 .\end{array}$ & $\begin{array}{c}\text { Estudo transversal e } \\
\text { retrospectivo }\end{array}$ & $\begin{array}{c}\text { Santa Catarina - } \\
\text { Criciúma, Içara, } \\
\text { Lauro Muller, } \\
\text { Morro da } \\
\text { Fumaça, Nova } \\
\text { Veneza, } \\
\text { Siderópolis, } \\
\text { Urussanga, } \\
\text { Forquilhinha, } \\
\text { Cocal do Sul, } \\
\text { Treviso e Orleans }\end{array}$ & $\begin{array}{l}\text { Participaram dessa pesquisa } 54 \text { pacientes, a maioria era do sexo feminino }(57,4 \%) \text {, brancos }(79,6 \%) \text {, } \\
\text { residiam na zona urbana }(81,5 \%) \text {, eram casos novos }(81,5 \%) \text { e tinham idade média de } 43,3 \text { anos, sendo } \\
\text { a mínima de } 12 \text { anos e máxima de } 83 \text { anos, a faixa etária mais acometida pela doença estava entre } 30 \text { a } \\
39 \text { anos. Verificou-se que } 43,2 \% \text { dos pacientes não tinham lesão, distribuição semelhante entre as formas } \\
\text { clínicas tuberculóide e virchowiana ( } 27,8 \%) \text {. No sexo feminino houve prevalência das formas dimorfa e } \\
\text { indeterminda }(28,5 \%) \text { e a paucibacilares }(53,3 \%) \text {, e no sexo masculino a virchowiana }(39,1 \%) \text { e a } \\
\text { multibacilar }(50,9 \%) \text {. A detecção dos casos novos ocorreu por encaminhamento }(61,1 \%) \text { e no momento } \\
\text { do diagnóstico, a maioria dos casos não apresentava lesões cutâneas }(44,4 \%) \text { e tinham grau zero de } \\
\text { incapacidade (50\%). }\end{array}$ \\
\hline $\begin{array}{l}\text { Queiroz et al., } \\
\quad 2015\end{array}$ & $\begin{array}{l}\text { Identificar o perfil clínico e } \\
\text { epidemiológico de pacientes em } \\
\text { tratamento para reações hansênicas. }\end{array}$ & $\begin{array}{l}\text { Estudo quantitativo, } \\
\quad \text { descritivo, } \\
\text { transversal }\end{array}$ & Mossoró- RN & $\begin{array}{l}\text { As formas clínicas em que os estados reacionais aconteceram com maior frequência foram a virchowiana } \\
(55,74 \%) \text { e a dimorfa ( } 32,79 \%) \text {, sendo que } 91,80 \% \text { eram multibacilares. Durante o diagnóstico, } 52,45 \% \\
\text { dos pacientes já apresentavam algum grau de incapacidade física instalada (Grau I: } 37,70 \% \text { e Grau II: } \\
14,75 \% \text { e no momento de realização do estudo, } 70,49 \% \text { dos pacientes apresentaram algum grau de } \\
\text { incapacidade física (Grau I: } 44,26 \% \text { e Grau II: } 26,23 \% \text { ). Ao término do tratamento, } 40,98 \% \text { dos pacientes } \\
\text { apresentavam alguma incapacidade física (Grau I: } 22,95 \% \text { e Grau II: } 18,03 \% \text { ). Outrossim, } 32,79 \% \text { dos } \\
\text { pacientes apresentaram apenas manchas hipocrômicas ou hiperemiadas no corpo e 16,39\% apresentaram } \\
\text { condições múltiplas. }\end{array}$ \\
\hline Pinho et al., 2015 & $\begin{array}{l}\text { Examinar a porcentagem de contatos com } \\
\text { índices positivos } \\
\text { para DNA de Mycobacterium leprae e } \\
\text { antígeno fenol-glicolipídeo-1 (PGL-1). }\end{array}$ & - & Maranhão & $\begin{array}{l}\text { A idade média dos pacientes foi de } 37(\mathrm{dp}= \pm 17,5 \text { anos). Além disso, os números de casos multibacilares } \\
(61 \%) \text { e pacientes limítrofes-tuberculóides }(32 \%) \text { foram significativos. A idade média dos contatos } \\
\text { intradomiciliares de casos da forma multibacilar }(70 \%) \text { era } 27,5(\mathrm{dp}= \pm 18,3 \text { anos). }\end{array}$ \\
\hline Alves et al., 2017 & $\begin{array}{l}\text { Analisar o perfil epidemiológico da } \\
\text { hanseníase no estado do Piauí, Brasil. }\end{array}$ & $\begin{array}{l}\text { Estudo transversal } \\
\text { com coleta } \\
\text { retrospectiva }\end{array}$ & Piauí & $\begin{array}{l}\text { A pesquisa constatou que houve } 13.787 \text { casos de hanseníase no período entre } 2005 \text { e } 2014 \text {, com } \\
\text { diminuição dos índices de avaliação sobre o grau de incapacidade física no diagnóstico, sendo o maior } \\
\text { percentual em } 2005(96,1 \%) \text {. As maiores taxas de cura ocorreram nos anos de } 2007 \text { (87,7\%) e } 2008 \\
(87,8 \%) \text {. }\end{array}$ \\
\hline
\end{tabular}




\begin{tabular}{|c|c|c|c|c|}
\hline $\begin{array}{c}\text { Gaudenci et al., } \\
2018\end{array}$ & $\begin{array}{l}\text { Evidenciar as condições socioeconômicas } \\
\text { e demográficas, clínicas, características, } \\
\text { trajetória da doença e grau de incapacidade } \\
\text { em pessoas com hanseníase. }\end{array}$ & $\begin{array}{l}\text { Estudo } \\
\text { observacional, } \\
\text { quantitativo e } \\
\text { transversal }\end{array}$ & Uberaba - MG & $\begin{array}{l}\text { Dos } 32 \text { pacientes em que foram realizadas a análise dos prontuários, a idade variou de } 22 \text { a } 89 \text { anos de } \\
\text { idade, com média de } 49,28 \text { anos }(\mathrm{dp}= \pm 16,93) \text {. A maioria possuía renda familiar entre um a três salários } \\
\text { mínimos }(43,8 \%) \text {, eram aposentados ou donas(os) de casa }(15,6 \%) \text {, sexo masculino }(59,4 \%) \text {, raça/ cor } \\
\text { preta ( }(46,9 \%) \text {, relataram manchas na pele como primeiro sintoma percebido }(43,8 \%) \text { e procuraram uma } \\
\text { Unidade Básica de Saúde }(53,1 \%) \text {, sendo que o tempo entre o surgimento dos primeiros sintomas e início } \\
\text { do tratamento foi de } 10 \text { a } 12 \text { meses e o tempo médio para o início do tratamento foi de } 4,54 \text { dias. As } \\
\text { pessoas que realizaram tratamento para cepa multibacilar }(84,4 \%) \text {, tinham uma média de } 2,44 \text { pessoas } \\
\text { como contatos, ainda que apenas } 1,75 \text { tenham sido examinadas. Apresentavam deficiência física de grau } \\
1 \text { ( } 37,5 \%) \text {, ainda que não tenham sido avaliados quanto aos aspectos físicos e deficiências (25\%). } \\
\text { Verificou-se que quanto menor a escolaridade, renda familiar e economicamente ativo maior o grau de } \\
\text { deficiência que também aumentou com a idade. }\end{array}$ \\
\hline Melo et al., 2020 & $\begin{array}{l}\text { O objetivo do estudo foi descrever os } \\
\text { aspectos sociodemográficos e clínicos } \\
\text { características dos pacientes com ENH } \\
\text { atendidos em um hospital terciário do } \\
\text { Piauí, Brasil, classificando } \\
\text { eles de acordo com o EESS }\end{array}$ & $\begin{array}{l}\text { Um estudo } \\
\text { observacional, } \\
\text { transversal e } \\
\text { descritivo }\end{array}$ & Piauí & $\begin{array}{l}\text { Em relação à população avaliada, houve maior prevalência no sexo masculino (65,4\%), faixa etária entre } \\
31 \text { e } 49 \text { anos ( } 42,3 \%) \text {, ensino primário incompleto }(50 \%) \text {, residiam em área urbana }(69,2 \%) \text {, } \\
\text { apresentaram a forma virchowiana (65\%) e tinha uma história de Eritema nodoso }(96,2 \%) \text {. }\end{array}$ \\
\hline Santos et al., 2020 & $\begin{array}{l}\text { Avaliar as características epidemiológicas } \\
\text { e tendência de novos casos de hanseníase } \\
\text { em menores de } 15 \text { anos no estado da } \\
\text { Bahia, Brasil, entre } 2007 \text { e } 2017 \text {. }\end{array}$ & $\begin{array}{l}\text { Estudo quantitativo, } \\
\text { transversal e } \\
\text { descritivo de casos }\end{array}$ & Bahia & $\begin{array}{l}\text { No recorte temporal em estudo, foram analisados } 2.298 \text { casos novos de hanseníase em menores de } 15 \\
\text { anos. A taxa de detecção da doença demonstrou tendência decrescente, sendo que a maioria era } \\
\text { paucibacilar }(63,27 \%) \text {. Em relação à predominância de casos novos e porcentagem de paucibacilares, } \\
\text { destacam-se, respectivamente, o sexo feminino }(53,16 \%) \text {, ensino fundamental incompleto }(66,02 \%) \text {, } \\
\text { pardos }(63,34 \%) \text {, residiam na zona urbana }(77,79 \%) \text {, forma clínica tuberculóide }(26,68 \%) \text { e } \\
\text { indeterminada }(26,07 \%) \text {, incapacidade grau zero }(73,72 \%) \text { e detecção por meio de encaminhamento } \\
(36,42 \%) \text {. Quanto à proporção de cura, a análise temporal revelou-se com tendência decrescente. }\end{array}$ \\
\hline Araújo et al., 2021 & $\begin{array}{c}\text { Analisar o quadro epidemiológico } \\
\text { tendências de perfil e hanseníase de } 2005 \text { a } \\
2018 \text {, além } \\
\text { das características clínicas dos pacientes. }\end{array}$ & Coorte retrospectivo & $\begin{array}{l}\text { Cruzeiro do Sul - } \\
\text { AC }\end{array}$ & $\begin{array}{l}\text { Dos } 422 \text { casos de hanseníase, a maioria era do sexo masculino }(67,3 \%) \text {, cor/ raça preta }(85,1 \%) \text {, idade } \\
\text { entre } 30 \text { a } 59 \text { anos }(45,1 \%) \text {, não referiram escolaridade }(37 \%) \text { e ocupação }(38,2 \%) \text {. Do total de casos } \\
\text { novos, } 45(10,8 \%) \text { eram crianças }(<15 \text { anos). O exame de contato e a demanda espontânea foram as mais } \\
\text { comuns formas de detecção entre os pacientes notificados, sendo que entre os casos detectados, as } \\
\text { maiores porcentagens de nenhuma deficiência física (grau } 0 \text { ) ocorreu entre aqueles com quadro clínico } \\
\text { limítrofe }(47,6 \%) \text { e tuberculóide }(29,3 \%) \text {. Para a forma clínica virchowiana, } 50 \% \text { dos casos foram } \\
\text { classificados como grau } 2 \text {, sendo que os casos mais graves foram mais comuns entre os homens. }\end{array}$ \\
\hline
\end{tabular}

Fonte: Elaborado pelos autores (2021). 


\section{Discussão}

Os estudos apontam que a maioria dos casos de hanseníase estavam na faixa etária entre 30 a 59 anos (Araújo et al., 2021; Melo et al., 2020; Melão et al., 2011; Miranzi et al., 2010), cuja idade média foi de 37 anos (Pinho et al., 2015), 43,3 anos (Melão et al., 2011) e 49,28 anos (Gaudenci et al., 2018), reforçando que o acometimento acontece entre aqueles que estão na fase economicamente ativa. Além desses dados, alguns trabalhos trouxeram a idade mínima e máxima dos pacientes atendidos, cuja variação ocorreu entre 12 e 89 anos (Gaudenci et al., 2018; Melão et al., 2011), sendo que a detecção em menores de 15 anos vem diminuindo no estado da Bahia (Santos et al., 2020), ainda represente 10,5\% dos casos (Araújo et al., 2021).

As pesquisas mostraram predomínio do sexo masculino (Araújo et al., 2021; Gaudenci et al., 2018; Melo et al., 2020; Miranzi et al., 2010), ainda que o feminino tenha sido identificado como predominante em outros estudos (Melão et al., 2011; Santos et al., 2020), o que pode ser resultado das campanhas destinadas à saúde da mulher, bem como comportamentos destinados ao autocuidado e preocupação com a estética, além do subdiagnóstico no sexo masculino nestas regiões (Melão et al., 2011; Santos et al., 2020) e barreiras na implementação de ações efetivas de prevenção e controle da doença (Queiroz et al., 2015).

Para a variável raça/ cor, também há uma divergência entre os autores, o que pode estar relacionado com as características da população nas diferentes regiões geográficas onde houve o processo de miscigenação refletindo no adoecimento pela hanseníase (Araújo et al., 2021; Gaudenci et al., 2018; Melão et al., 2011; Miranzi et al., 2010; Santos et al., 2020).

Estes pacientes residiam majoritariamente na zona urbana (Melão et al., 2011; Melo et al., 2020; Miranzi et al., 2010; Santos et al., 2020) e dentre aqueles que abordavam sobre a escolaridade, três evidenciaram ensino primário incompleto (Melo et al., 2020), de quatro a sete anos (Miranzi et al., 2010) e ensino primário incompleto (Santos et al., 2020), embora nem todos os participantes tenham referido sua escolaridade (Araújo et al., 2021).

A baixa escolaridade implica diretamente no reconhecimento tardio dos sinais e sintomas, bem como na compreensão das diretrizes da doença, o que retarda a procura pelo serviço de saúde precocemente com o início dos sinais e sintomas e, consequentemente, resulta em maior risco de sequelas e desafios diante da complexidade destes casos (Melão et al., 2011; Queiroz et al., 2015).

Assim, é essencial que as unidades de saúde invistam em estratégias educacionais para detecção nos territórios (Alves et al., 2017) e a inclusão com corresponsabilização dos pacientes no seu autocuidado (Queiroz et al., 2015), as quais contribuirão na adesão do indivíduo à terapia.

No que se refere à ocupação, a maioria era aposentados ou donas de casa (Gaudenci et al., 2018), embora esta não seja uma variável abordada em outros estudos (Araújo et al., 2021; Gaudenci et al., 2018), e em alguns com o foco na renda familiar, com variação de um a três salários mínimos, os quais podem contribuir no acesso aos serviços de saúde (Gaudenci et al., 2018).

Em relação ao perfil clínico da doença, em contraste com as recomendações da Organização Mundial da Saúde (OMS) que preconiza a busca ativa de casos, os estudos indicaram uma prevalência, em números absolutos, de casos novos oriundos majoritariamente de encaminhamentos (Melão et al., 2011; Miranzi et al., 2010; Santos et al., 2020). Isso indica a necessidade de implementação de mais ações destinadas à busca ativa de casos e investigação de contato nas unidades da Atenção Primária à Saúde para que ocorra melhoria no diagnóstico precoce de casos e controle da doença (Araújo et al., 2021), considerando que $96,2 \%$ das residências de pessoas que tiveram casos diagnosticados com hanseníase, residiam dois ou mais indivíduos (Gaudenci et al., 2018).

Quanto à forma clínica, devido à complexidade da doença, identificou-se os tipos limítrofe (Araújo et al., 2021), dimórfica (Miranzi et al., 2010), não classificados (Gaudenci et al., 2018) e indeterminada (Santos et al., 2020), sendo que a prevenção e o rastreamento na comunidade são essenciais e deveriam ser prioritárias no planejamento e execução das ações de 
vigilância (Miranzi et al., 2010), a fim de contribuir na diminuição do coeficiente de incidência (Alves et al., 2017) e interrupção da cadeia de transmissão, principalmente quando mais da metade dos casos eram paucibacilar (Miranzi et al., 2010; Santos et al., 2020) em crianças na Região Norte do país (Santos et al., 2020).

Ressalta-se ainda o predomínio do grau zero de incapacidade física, o que indica a detecção precoce destes casos (Araújo et al., 2021; Melão et al., 2011; Santos et al., 2020), ainda que não seja uma realidade em todos os cenários, onde encontrou grau 1 e 2 (Gaudenci et al., 2018), evidenciando desafios nas ações de prevenção em algumas regiões do Brasil (Queiroz et al., 2015).

Sobre as reações hansêmicas, a literatura aponta que poderiam evoluir para uma possível incapacidade física (Melo et al., 2020), mesmo após o término do tratamento, desencadeada por alguma condição reativa (Queiroz et al., 2015).

\section{Conclusão}

O estudo permitiu identificar o perfil majoritário dos pacientes acometidos pela hanseníase no Brasil, o que possibilita compreender o cenário mesmo diante da diversidade nas cinco regiões do país, reforçando sobre a importância da articulação dos serviços que constituem a RAS para manejo adequado dos casos e efetividade das ações de vigilância em saúde, principalmente quando a detecção ocorre nos serviços especializados em detrimento da Atenção Primária à Saúde. Além disso, sugere-se que outros estudos sejam desenvolvidos para compreender a percepção destes pacientes após o adoecimento pela hanseníase, identificando o percurso, dificuldades e ações exitosas vivenciadas por estes.

Como limitação desta revisão, destaca-se o uso de três bases de dados, o que diminuiu a outras informações que poderiam contribuir no conhecimento do panorama da hanseníase no sistema de saúde brasileiro, considerando ainda a concentração de estudos em locais específicos, tais como o Nordeste.

\section{Referências}

Alves, E. S., Oliveira, L. B. de, Araújo, T. E. de, Melo, I. V. de, Araújo, R. de P. S., \& Marques, L. M. F. (2017). Perfil epidemiológico da hanseníase em um município do nordeste brasileiro: uma análise retrospectiva. Revista de Pesquisa: Cuidado é Fundamental Online, 9(3), 648-652. https://doi.org/10.9789/21755361.2017.v9i3.648-652.

Araújo, F. A., Abreu, L. C., Laporta, G. Z., Santos, V. S., Moreira, J. G. V., \& Grumach, A. S. (2021). Hanseniasis in the municipality of Western Amazon (Acre, Brazil): are we far from the goal of the World Health Organization? Hansen and Western Amazon. The Brazilian Journal of Infectious Diseases, 25(1), 101042. https://doi.org/10.1016/j.bjid.2020.11.007.

Brasil. Ministério da Saúde. (2021a). Guia de Vigilância em Saúde. (5a ed.), Ministério da Saúde.

Brasil. Ministério da Saúde. (2021b). Boletim Epidemiológico de Hanseníase 2021. https://www.gov.br/saude/ptbr/media/pdf/2021/setembro/8/boletim_hanseniase_internet_-2.pdf

Brasil. Ministério da Saúde. (2019). Estratégia de Enfretamento da Hanseníase 2019/2022. https://antigo.saude.gov.br/images/pdf/2020/May/22/estr--tegianacional-de-hanseniase-2019-2022-web.pdf

Brasil. Ministério da Saúde. (2017). Guia prático sobre a hanseníase. Disponível em: https://dvs.portovelho.ro.gov.br/uploads/editor/files/SEMUSA/GuiaPratico-de-Hanseniase.pdf

Brasil. Ministério da Saúde. (2015). Eliminar a hanseníase é possível: um guia prático para os municípios. https://bvsms.saude.gov.br/bvs/publicacoes/eliminar_hanseniase_possivel_versao_preliminar.pdf

Brasil. Ministério da Saúde. (2008). Vigilância em Saúde: situação epidemiológica da hanseníase no Brasil. https://bvsms.saude.gov.br/bvs/publicacoes/vigilancia_saude_situacao_hanseniase.pdf

Gaudenci, E. M., Nardelli, G. G., Carleto, C. T., \& Pedrosa, L. A. K. (2018). Sociodemographic and clinical profile of Hansen's disease patients in a specialized center. Bioscience Journal, 34(6), 1765-1774. https://doi.org/10.14393/bj-v34n6a2018-39471.

Melão, S., Blanco, L. F. de O., Mounzer, N., Veronezi, C. C. D., \& Simões, P. W. T. de A. (2011). Perfil epidemiológico dos pacientes com hanseníase no extremo sul de Santa Catarina, no período de 2001 a 2007. Revista da Sociedade Brasileira de Medicina Tropical, 44(1), 79-84. https://doi.org/10.1590/s003786822011000100018 .

Melo, C. B. De, Sá, B. D. S. De, Costa, F. A. C., \& Sarno, E. N. (2020). Epidemiological profile and severity of erythema nodosum leprosum in Brazil: a crosssectional study. International Journal of Dermatology, 59(7), 856-861. https://doi.org/10.1111/ijd.14895. 
Research, Society and Development, v. 10, n. 16, e289101623741, 2021

(CC BY 4.0) | ISSN 2525-3409 | DOI: http://dx.doi.org/10.33448/rsd-v10i16.23741

Miranzi, S. de S. C., Pereira, L. H. de M., \& Nunes, A. A. (2010). Perfil epidemiológico da hanseníase em um município brasileiro, no período de 2000 a 2006. Revista da Sociedade Brasileira de Medicina Tropical, 43(1), 62-67. https://doi.org/10.1590/s0037-86822010000100014.

Ouzzani, M., Hammady H., Fedorowicz, Z., \& Elmagarmid, A. (2016). Rayyan - a web and mobile app for systematic reviews. Syst Revi., 5(1), 1-10. Disponível em: https://www.ncbi.nlm.nih.gov/pmc/articles/PMC5139140/pdf/13643

Pinho, J. D., Rivas, P. M. S., Mendes, M. B. P., Soares, R. E. P., Costa, G. C., Nascimento, F. R. F., Paiva, M. F. L., Aquino, D. M. C., Figueireido, I. A., Santos, A. M., \& Pereira, S. R. F. (2015). Presence of Mycobacterium leprae DNA and PGL-1 antigen in household contacts of leprosy patients from a hyperendemic area in Brazil. Genetics and Molecular Research, 14(4), 14479-14487. https://doi.org/10.4238/2015.

Queiroz, T. A., Carvalho, F. P. B. de, Simpson, C. A., Fernandes, A. C. L., Figueirêdo, D. L. de A., \& Knackfuss, M. I. (2015). Perfil clínico e epidemiológico de pacientes em reação hansênica. Revista Gaúcha de Enfermagem, 36(spe), 185-191. https://doi.org/10.1590/1983-1447.2015.esp.57405.

Santos, Á. N., Costa, A. K. A. N., Souza, J. É. R. de, Alves, K. A. N., Oliveira, K. P. M. M. de, \& Pereira, Z. B. (2020). Perfil epidemiológico e tendência da hanseníase em menores de 15 anos. Revista da Escola de Enfermagem da USP, 54, e03659. https://doi.org/https://doi.org/10.1590/S1980-220X2019016803659.

Santos, R. F. da S., Silva, N. L. B., Santos, D. C. M. dos, D’Azevedo, S. S. P., \& Oliveira, D. A. L. (2018). A organização da rede de atenção às pessoas atingidas pela Hanseníase no município do Recife. Revista Enfermagem Digital Cuidado e Promoção da Saúde, 3(2), 36-41. https://doi.org/10.5935/2446-5682.20180006.

The Joanna Briggs Institute. (2014). Joanna Briggs Institute Reviewers' Manual: The Systematic Review of Economic Evaluation Evidence. https://nursing.lsuhsc.edu/JBI/docs/ReviewersManuals/Economic.pdf

WHO. (2021). Global leprosy (Hansen disease) update, 2020: impact of COVID-19 on the global leprosy control. https://www.who.int/publications/i/item/whower9636-421-444.

WHO. (2016). Estratégia Global para Hanseníase 2016-2020: Aceleração rumo a um mundo sem hanseníase 2016-2020. https://apps.who.int/iris/bitstream/handle/10665/208824/9789290225201-pt.pdf. 